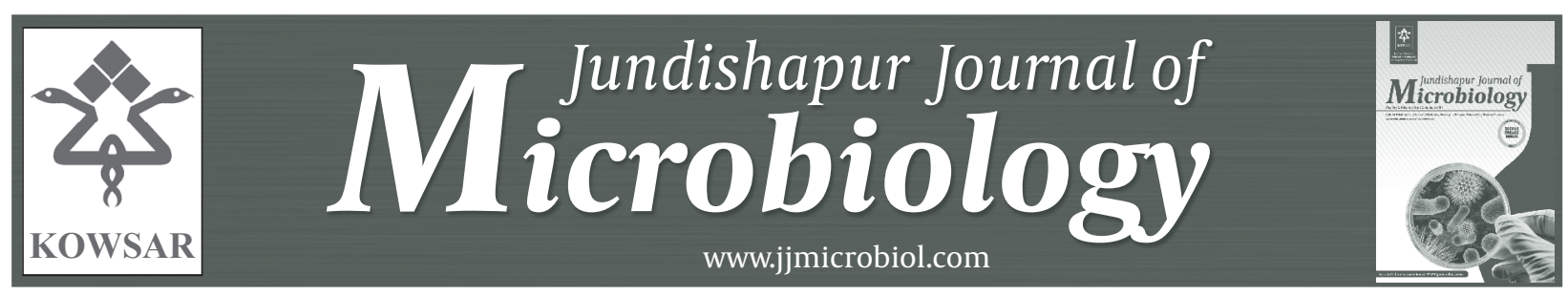

\title{
Antibiotic Resistance Pattern of Methicillin Resistant and Methicillin Sensi- tive Staphylococcus aureus Isolates in Tehran, Iran
}

\author{
Fateh Rahimi ${ }^{1}$, Majid Bouzari ${ }^{1^{*}}$, Mohammad Katouli ${ }^{2}$, Mohammad Reza Pourshafie ${ }^{3^{*}}$ \\ ${ }^{1}$ Department of Biology, Faculty of Science, University of Isfahan, Isfahan, IR Iran \\ ${ }^{2}$ Faculty of Science, Health and Education, University of the Sunshine Coast, Sunshine Coast, Australia \\ ${ }^{3}$ Department of Bacteriology, Pasteur Institute of Iran, Tehran, IR Iran \\ * Corresponding author: Majid Bouzari, Department of Biology, Faculty of Science, University of Isfahan, Hezarjereeb Street, Isfahan 81746-73441, IR Iran. \\ Tel.: +98-3117932459, Fax: +98-3117932456, E-mail: bouzari@sci.ui.ac.ir; Mohammad Reza Pourshafie, Department of Bacteriology, Pasteur Institute of Iran, \\ Tehran, 13164, IR Iran.Tel.: +98-2166405535, Fax: +98-2166405535, E-mail: pour@pasteur.ac.ir
}

\begin{abstract}
A B S T R A C T
Background: Staphylococcus aureus is associated with different infections ranging from skin and soft tissue infections to endocarditis and fatal pneumonia. S. aureus is still the most common bacterial species isolated from inpatient specimens and the second most common from outpatient specimens. Today, methicillin resistant S. aureus (MRSA) isolates are present in the hospitals of most countries and are often resistant to several antibiotics.

Objectives: This study was conducted from 2007 to 2011 to detect prevalence and antibiotic resistance patterns among MRSA and methicillin sensitive S. aureus (MSSA) isolated from hospitals in Tehran, Iran.

Materials and Methods: Totally 726 isolates of S. aureus were collected from three referral hospitals in Tehran. All isolates were identified at the species level by standard biochemical tests. Susceptibility to eighteen antibiotics was determined by disc diffusion method. Then oxacillin and vancomycin minimum inhibitory concentration (MIC) of resistant isolates was also determined using Etest. mecA gene was detected using specific primers.

Results: A total of 216(30\%) strains were found to be MRSA isolates. The highest antibiotic resistance was to penicillin, clindamycin, tobramycin and tetracycline respectively. Ninety three and 61\% of MRSA and MSSA isolates were multidrug resistant(MDR) respectively. However, no strain was resistant to vancomycin, synercid, linezolid and chloramphenicol. Sixty nine percent of MRSA isolates showed high level of resistance to oxacillin (MIC $\geq 256 \mu \mathrm{g} / \mathrm{mL}$ ). mecA gene was detected among all MRSA isolates.

Conclusions: Although the frequency of MRSA isolates in the current study was low, resistance to other antibiotics was high and most of the isolates were found to be MDR. Regular surveillance of hospital-associated infections and monitoring of their antibiotic sensitivity patterns are required to reduce MRSA prevalence. High frequency of MDR isolates of S. aureus could be considered as an urgent warning for public health.
\end{abstract}

Keywords: Staphylococcus aureus; Vancomycin; Iran

Copyright (c) 2013, Kowsar Corp.; Published by Kowsar Corp.

Article type: Research Article; Received: 15 Mar 2012, Revised: 07 May 2012, Accepted: 21 May 2012; DOI:10.5812/jjm.4896

Implication for health policy/practice/research/medical education:

This study was conducted to indicate the prevalence of methicillin resistant Staphylococcus aureus (MRSA) isolates in Tehran hospitals and also to highlight the differences of antibiotic resistance patterns among MRSA and methicillin sensitive S. aureus (MSSA) isolates.

Please cite this paper as:

Rahimi F, Bouzari M, Katouli M, Pourshafie MR. Antibiotic Resistance Pattern of Methicillin Resistant and Methicillin Sensitive Staphylococcus aureus Isolates in Tehran, Iran. Jundishapur J Microbiol. 2013;6(2):144-149. DOI: 10.5812/jjm.4896

Copyright @ 2013 Ahvaz Jundishapur University of Medical Sciences; Published by Kowsar Corp.

This is an Open Access article distributed under the terms of the Creative Commons Attribution License (http://creativecommons.org/licenses/by/3.0), which permits unrestricted use, distribution, and reproduction in any medium, provided the original work is properly cited. 


\section{Background}

Staphylococcus aureus is a hospital and communityacquired pathogen that causes a broad spectrum of diseases, ranging from skin and soft tissue infections to endocarditis and fatal pneumonia. This pathogenicity is associated with different enzymes and toxins such as enterotoxins, exfoliative toxin, toxic shock syndrome toxin, and Panton-Valentine leucocidin (PVL) $(1,2)$.

This bacterium has the remarkable ability to adapt to different antibiotics and now with the emergence of multidrug resistant (MDR) bacteria, S. aureus is a warning for public health $(1,3)$. Methicillin-resistant S. aureus (MRSA) strains are able to grow in the presence of methicillin, oxacillin and nafcillin (4).

Methicillin resistance in S. aureus was initially detected in Europe in the 1960s, only one year after the introduction of methicillin $(5,6)$. Today, MRSA isolates are found not only in the hospitals of most countries but also in communities and are often resistant to several antibiotics (7). Clinical infections are most common in patients in hospital intensive care units, nursing homes, and other chronic care facilities; however, MRSAs are emerging as an important community acquired pathogen as well. Although there are some reports on the prevalence of vancomycin resistant S. aureus (VRSA) and vancomycin intermediate $S$. aureus (VISA), most MRSA isolates are susceptible to vancomycin and teicoplanin; therefore resistance increase to these antibiotics results in the limitation of treatment options and also the requirement of a new class of antibiotics $(8,9)$.

$S$. aureus is the most important cause of infection among hospitalized patients and also the second cause of infection among outpatients (10). Today, S. aureus is the leading cause of nosocomial pneumonia and the second leading cause of bloodstream infections in the world (11). MRSA is also dominant in intensive care unit (ICU) of hospitals in most parts of the world (12).

The emergence of novel community-acquired MRSA (CA-MRSA) strains has complicated the control and prevention of infections caused by MRSA isolates. The genetic and phenotypic properties of these CA-MRSA isolates are completely different from hospital acquired MRSA (HAMRSA) isolates $(13,14)$. These differences in the structure of CA and HA-MRSA strains results in antibiotic resistance patterns differences of isolates influencing the hospital and community environments (15). Increasing rate of CAMRSA isolates recovered from patients in different parts of the world, suggests that these strains are displacing among nosocomial MRSA strains and might behave similar to traditional nosocomial MRSA strains with respect to mortality and other various clinical outcomes $(13,16)$.

\section{Objectives}

There are several reports focusing on MRSA isolates prevalence in different parts of Iran. But, the current study aimed to analyze the prevalence and also antibiotic susceptibility patterns of MRSA and methicillin sensitive S. aureus (MSSA) isolated from three referral hospitals in Tehran.

\section{Materials and Methods}

\subsection{Sample Collection}

A total of 726 isolates of S. aureus were collected during 2007 to 2011 from three referral hospitals in different parts of Tehran including three university hospitals (A-C) in south, north and center of Tehran with the associated number of isolates 194, 321 and 211 respectively.

\subsection{Isolation and Phenotyping of S. aureus}

All of the isolates were confirmed as Staphylococcus genus by different biochemical tests such as Gram staining, catalase and oxidase (17). Catalase, Gram positive and oxidase negative isolates were defined as Staphylococcus. Isolates indicating Staphylococcus characteristics were further analyzed by fermentation in manitol salt agar medium, DNase and coagulase tests (17). All S. aureus were DNase and coagulase positive and fermented manitol. $S$. aureus ATCC 29213 and S. epidermidis ATCC 35984 were used as negative and positive controls.

\subsection{Antimicrobial Susceptibility Testing}

Susceptibility of $S$. aureus isolates to oxacillin $(1 \mu \mathrm{g}), \mathrm{Ka}-$ namycin $(30 \mu \mathrm{g})$, amikacin $(30 \mu \mathrm{g})$, penicillin $(5 \mu \mathrm{g})$, fusidic acid $(10 \mu \mathrm{g})$, minocycline $(30 \mu \mathrm{g})$, erythromycin (15 $\mu \mathrm{g})$, clindamycin $(2 \mu \mathrm{g})$, tobramycin $(10 \mu \mathrm{g})$, rifampicin $(2 \mu \mathrm{g})$, nitrofurantoin $(50 \mu \mathrm{g})$, sulphamethoxazole-trimethoprime $(1.25-23.75 \mu \mathrm{g})$, linezolid $(10 \mu \mathrm{g})$, synercid $(15 \mu \mathrm{g})$, chloramphenicol $(30 \mu \mathrm{g})$, ciprofloxacin $(30 \mu \mathrm{g})$, gentamicin $(10 \mu \mathrm{g})$ and tetracycline $(30 \mu \mathrm{g})$ (MAST Group, Merseyside, United Kingdom) was determined by disc diffusion method according to the guidelines of Clinical and Laboratory Standards Institute (CLSI) (18). All methicillin resistant strains were collected and MICs of oxacillin and vancomycin among MRSA isolates were determined by Etest (AB, Biomerieux, Marcy l'Etoile, France) according to the manufacturer's instructions and were repeatedby CLSI guidelines (19).

\subsection{DNA Extraction}

DNA extraction was done by High Pure PCR Template Preparation Kit (Roche, Mannheim, Germany) according to manufacturer's instructions with some modifications. The concentrations of all extracted DNAs were determined by Nanodrop 1000 (NanoDrop, Wilmington, USA). One micro liter of each DNA was used as template in PCR reaction. 


\subsection{PCR}

PCR primers specific for mecA gene (mecA1: GTAGAAATGACTGAACGTCCGATAA and mecA2: CCAATTCCACATTGTTTCGGTCTAA) were selected from Jonas et al. (20) and synthesized by Tib-Molbiol (Berlin, Germany). The PCR mixture containing; 10X PCR buffer, taq DNA polymerase

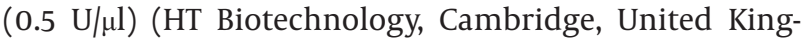
dom), each primer $(1.6 \mu \mathrm{M}), \mathrm{MgCl} 2(1.2 \mu \mathrm{M})$ and each dNTP $(0.64 \mu \mathrm{M})$. The PCR cycles for the isolates were as follows: an initial denaturation at $94^{\circ} \mathrm{C}$ for $5 \mathrm{~min}$, with 30 cycles of denaturation at $94^{\circ} \mathrm{C}$ for $15 \mathrm{~s}$, annealing at $61^{\circ} \mathrm{C}$ for $15 \mathrm{~s}$ and elongation at $72^{\circ} \mathrm{C}$ for 30 s and final extension at $72^{\circ} \mathrm{C}$ for 5 min (20). PCR products were electrophoresed on a $1.5 \%$ agarose gel in a $0.5 \mathrm{X}$ Tris-Borate-EDTA (TBE) buffer and stained in ethidium bromide.

\section{Results}

Totally, all suspected 726 isolates gathered from different hospitals were confirmed as $S$. aureus by standard biochemical tests. 216 isolates (29.7\%) were selected as MRSA and were analyzed more. As shown in Figure 1, more than $98 \%$, the highest level, of the S. aureus isolates were resistant to penicillin. All MRSA and MSSA isolates were susceptible to chloramphenicol, synercid and linezolid, and also more than $99 \%$ of the isolates were susceptible to nitrofurantoin and fusidic acid. Resistance to gentamicin, minocycline, SXT and rifampicin was less than $30 \%$. Resistance to clindamycin, tobramycin and tetracycline was higher than other antibiotics and more than $40 \%$ of total isolates were resistant to these three antibiotics.

Figure 1. Percentage of Antibiotic Resistance in S. aureus Strains.

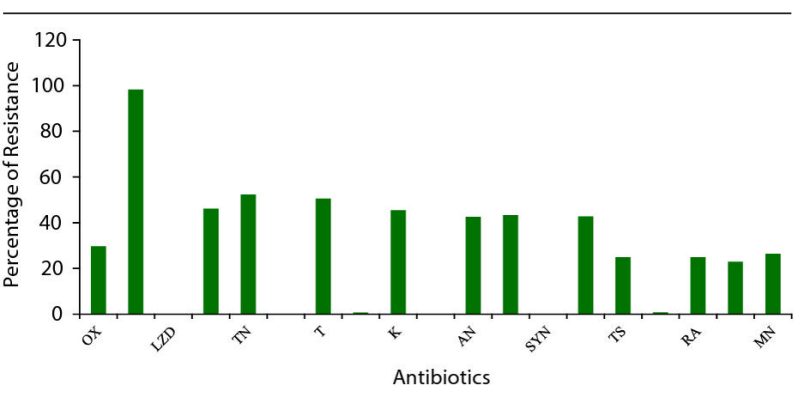

Abbreviations: OX, Oxacillin; P, Penicillin; LZD; Linezolid, CD; Clindamycin, TN; Tobramycin, C; Chloramphenicol; T, Tetracycline; NI, Nitrofurantoin; K, Kanamycin; VA,Vancomycin; AN, Amikacin; E, Erythromycin; SYN, Synercid; CIP, Ciprofloxacin; TS, Cotrimoxazole; FC, Fusidic Acid; RA, Rifampicin; GM, Gentamicin; MN, Minocycline

In antimicrobial susceptibility test of MRSA isolates, the number of bacteria resistant to penicillin, ciprofloxacin, tobramycin, kanamycin, erythromycin, clindamycin, tetracycline and amikacin were the highest (Table 1). None of the isolates were resistant to chloramphenicol, synercid and linezolid. Only 6\% of MRSA isolates were re- sistant to all antibiotics other than these three antibiotics. Seven percent of MRSA isolates were susceptible to all antibiotics except for penicillin.

Table 1. Frequency of Antibiotic Resistance of MRSA and MSSA Strains.

\begin{tabular}{lll}
\hline & MRSA, No. (\%) & MSSA, No.(\%) \\
\hline Oxacillin & $216(100)$ & $0(0)$ \\
\hline Penicillin & $216(100)$ & $498(98)$ \\
\hline Clindamycin & $163(75)$ & $172(34)$ \\
\hline Nitrofurantoin & $5(2)$ & $0(0)$ \\
\hline Tobramycin & $196(91)$ & $184(36)$ \\
\hline Tetracycline & $179(83)$ & $189(37)$ \\
\hline Chloramphenicol & $0(0)$ & $0(0)$ \\
\hline Fusidic Acid & $6(3)$ & $0(0)$ \\
\hline Kanamycin & $184(85)$ & $147(29)$ \\
\hline Amikacin & $181(84)$ & $129(25)$ \\
\hline Vancomycin & $0(0)$ & $0(0)$ \\
\hline Erythromycin & $201(93)$ & $114(22)$ \\
\hline Ciprofloxacin & $205(95)$ & $106(21)$ \\
\hline Synercid & $0(0)$ & $0(0)$ \\
\hline SXT & $136(63)$ & $45(9)$ \\
\hline Rifampicin & $147(68)$ & $42(8)$ \\
\hline Gentamicin & $127(59)$ & $40(8)$ \\
\hline Minocycline & $106(49)$ & $86(17)$ \\
\hline Linezolid & $0(0)$ & $0(0)$ \\
\hline
\end{tabular}

In the current study no vancomycin resistant MRSA strain could be isolated and the frequencies of vancomysin resistant S. aureus (VRSA) and vancomycin intermediate $S$. aureus (VISA) were zero.

According to the comparison of antibiotic resistance patterns among MRSA and MSSA isolates, as indicated in Table 1, 98\% of MSSA isolates were resistant to penicillin and it was the only antibiotic that most of the MSSA isolates were resistant to. In the case of clindamycin, tobramycin, tetracycline and amikasin more than 2.2 fold increase in resistance was observed among MRSA isolates in comparison to MSSA isolates. Moreover, in the case of amikacin this rate was increased to more than 3 fold. Interestingly, for erythromycin, ciprofloxacin, gentamicin and SXT this range varied from 4 to 7 fold. In MRSA isolates the rate of gentamicin resistance was $59 \%$, while in MSSA isolates it was only $8 \%$. Also for rifampicin, in MRSA isolates the rate of resistance was $68 \%$ but only $8 \%$ of MSSA isolates were resistant to this antibiotic. Seventy five percent of MRSA isolates were resistant to clyndamicin, while $34 \%$ of MSSA exhibited resistance to clindamycin. Not only MRSA isolates, but also MSSA isolates were susceptible to linezolid, synercid and chloramphenicol. Nitrofurantoin and fusidic acid were the two antibiotics 
that 98 and $97 \%$ of MRSA isolates exhibited susceptibility to them respectively, and also no resistance was observed among MSSA isolates to these antibiotics.

According to Centers for Disease Control (CDC), MDRs are defined as microorganisms, predominantly bacteria, that are resistant to one or more classes of antimicrobial agents (21). Therefore, in MRSA and MSSA isolates, 9 and 5 different multi drug resistance patterns were determined, respectively (Figure 2). In the current study only 93\% of MRSA isolates were resistant to at least three antibiotics, while $61 \%$ of MSSA isolates were resistant to at least two antibiotics. Here, only 6\% of MRSA isolates were resistant to 12 antibiotics, and 34\% were resistant to eleven antibiotics. In MSSA isolates, only $3 \%$ were susceptible to all antibiotics and $26 \%$ were resistant to one antibiotic. In one hospital, the frequency of the MRSA isolates was completely different in comparison to other hospitals. In hospitals A-C, the frequency of MRSA isolates were 8, 9 and 32\% respectively. The frequency of MRSAs isolated from different sources is shown in Table 2 . Thirty nine percent of MRSA isolates were from wounds. The lowest number of MRSA isolates was associated with ear and eye infections. The frequency of MRSA isolates were 19, 12, 10 and $8 \%$ in urine, sputum, CSF and nose cultures, respectively. Also, 5 and 5\% of MRSA isolates were associated with blood and abscess cultures, respectively.

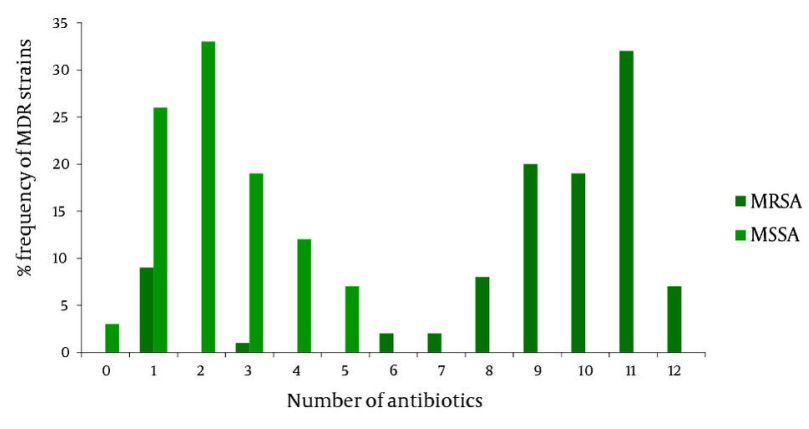

Figure 2. Frequency of MDR Patterns of MRSA and MSSA Isolates. Numbers Denote the Antibiotics That Strains Were Resistant.

Table 2. Distribution of MRSAs Isolated From Different Samples and Different Wards.

\begin{tabular}{|c|c|c|c|c|c|c|c|c|c|c|}
\hline & Gynecology & Surgery & ENT* & ICU* & Respiratory & Ophthalmology & Pediatrics & Oncology & Urology & Total \\
\hline Abcess & - & 1 & - & 2 & - & - & 4 & - & 4 & 11 \\
\hline Blood & 2 & 3 & - & 1 & - & - & 2 & 3 & - & 11 \\
\hline Ear & - & - & 3 & - & - & - & - & - & - & 3 \\
\hline Eye & - & - & - & - & - & 2 & - & - & - & 2 \\
\hline Nose & 5 & 2 & - & 3 & 3 & - & 4 & - & - & 17 \\
\hline Sputum & - & - & 1 & 3 & 15 & - & 1 & 6 & - & 26 \\
\hline CSF & 5 & 8 & - & 4 & - & - & - & 4 & - & 21 \\
\hline Urine & 11 & - & - & 8 & - & - & 6 & 4 & 12 & 41 \\
\hline Wound & 10 & 29 & - & 13 & - & - & 7 & 19 & 6 & 84 \\
\hline Total & 33 & 43 & 4 & 34 & 18 & 2 & 24 & 36 & 22 & 216 \\
\hline
\end{tabular}

Abbreviations: ENT, Ear, Nose and Throat; ICU, Intensive care unite; CSF, Cerebrospinal fluid

Considerable differences were observed when the distributions of MRSA isolates in different wards were compared (Table 2).Twenty , 15, 17, 16, 10, 11 and 8\% of MRSA isolates were recovered from surgery and operation, gynecology, oncology, intensive care, urology, pediatrics and respiratory units respectively. Only 2 and 1\% of the isolates were from ear, nose and throat (ENT), and ophthalmology unites, respectively.

Moreover, the frequency of MRSA isolates among elderly patients (61\%) was higher than the others. Thirty four and $5 \%$ of MRSA isolates were associated with adults and children, respectively. Also, in contrast to women (41\%), the frequency of methicillin resistance was the highest among S. aureus bacteria isolated from men (59\%).

MIC results using Etest showed that $100 \%$ of MRSA isolates were resistant to oxacillin (MIC $\geq 4 \mu \mathrm{g} / \mathrm{mL}$ ) (Figure 3). Eightyfivepercent of MRSA isolates were highly resistant to oxacillin (MIC $\geq 128 \mu \mathrm{g} / \mathrm{mL}$ ) and $4 \%$ of the isolates showed low resistance to oxacillin. The MIC ranges of 11\% of MRSA isolates varied from 24 to $96 \mu \mathrm{g} / \mathrm{mL}$. In PCR, mecA gene was detected in all 216 MRSA strains (Figure 4) which confirmed all isolates as MRSA.

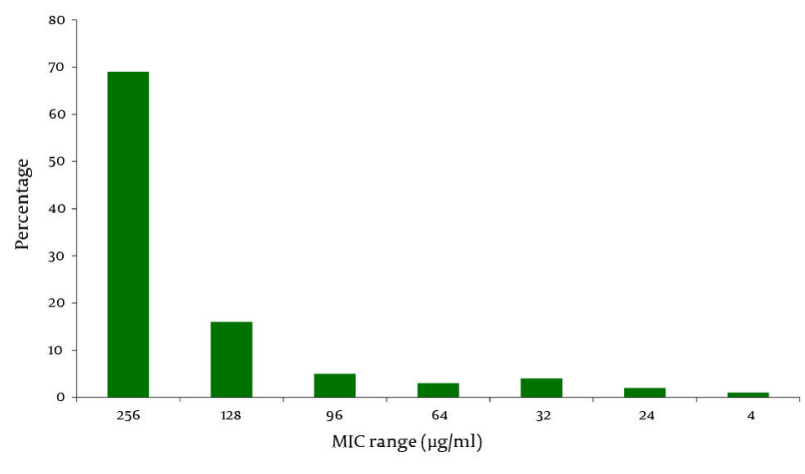

Figure 3. MICs Range in Different Isolates of MRSA. 


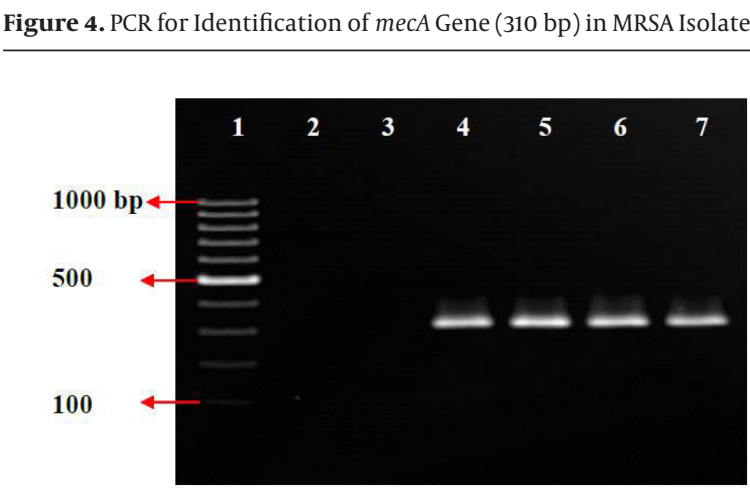

Column 1,1000 bp DNA ladder (Fermentas, Latvia). Columns 2-7, PCR reac tion: $2=$ none template control, $3=$ S. aureus ATCC 25923 (negative control), $4=$ S. aureus ATCC 29213 (positive control), 5-7=MRSA strains.

\section{Discussion}

Results of the current study indicated that the prevalence of MRSA isolates in three different hospitals in Tehran was $29.7 \%$. This rate of resistance was lower than the other reports from Iran $(42-90 \%)(8,22-25)$. However, this variation of MRSA isolates in different geographical regions of Iran might also be due to several other factors like efficacy of infection control practices, healthcare facilities and antibiotic usage that vary from hospital to hospital. MRSA infection has recently become a serious problem in anti-microbial chemotherapy. During the past four decades, MRSAs have spread throughout the world and have become highly endemic in many geographical areas. It has been suggested that due to the changing pattern of antibiotic resistance in S. aureus, it would be wiser to have a periodical surveillance of these changes every 3 to 4 years $(22,23)$.

The current study indicated that resistance to linezolid, synercid and chloramphenicol was low and they were the most effective antibiotics against MRSA isolates. This may be due to low consumption of these antibiotics in Iran. This could, in turn, suggest the lack of horizontal transfer of resistant genes from other bacterial species to MRSA. Although chloramphenicol is a very effective antibiotic against MRSA isolates in vitro, its prescription is influenced by different side effects, a fact that could explain low resistance frequency $(22,23)$. Although Linezolid and synercid are the most effective antibiotics against MRSA isolates, their high cost limits their consumption for treatment purposes.

Vancomycin is the last resort and drug of choice to treat infections caused by MRSA isolates in the world, therefore the emergence of resistance to vancomycin could be an urgent warning for public health. Current results were inconsistent with other studies in Iran which have reported high prevalence (7\%) of VRSA isolates (25). This might be due to using improper diagnostic methods. As recommended by CLSI, the standard methods for vancomycin resistance screening in S. aureus are, Etest, broth dilution and agar dilution; so the results of disk diffusion test is not reliable. Since, the incidence of nosocomial infections caused by MRSA isolates represents the measures for control and prevention in hospitals, the necessity to treat MRSA infections by vancomycin results in emergence of VRSA isolates. Therefore, this antibiotic should be prescribed with caution.

The pattern of antibiotic susceptibility of MSSA and MRSA isolates differed significantly. The MSSA isolates were susceptible to most of the antibiotics tested, although some resistance was observed to penicillin, tetracycline, tobramycin, and to some extent to clindamycin, amikacin and kanamycin, the antibiotics were often used to treat general infections. In contrast, in the case of MRSA, multiple drug resistance was common and only a few antibiotics were active against these isolates. MRSA strains were found to be more resistant to other antibiotics than MSSA strains. Significant difference $(P$ value $<0.05)$ was observed in case of erythromycin, ciprofloxacin, tobramycin, tetracycline, gentamicin and amikacin. A significant difference was found between sensitivity patterns of MRSA and those of MSSA isolates $(24,26,27)$.

Differences in the frequency of methicillin resistance among S. aureus bacteria isolated from various specimens might be due to prolonged antibiotic treatment of severely sick patients, which generally have longer hospital stays, resulting in enhanced selection pressure (24). Distributions of MRSA isolates were varied in different wards which partly reflected the fact that some patients, e.g., critically ill patients in ICUs, had a greater chance of becoming colonized or infected (28).

In conclusion, the accurate diagnosis of MRSA strains in hospitals, patients and health care workers is an important need. Also the dissemination of MRSA and MSSA strains with high resistance to different antibiotics in Tehran hospitals is a warning for public health. Accurate and continuous surveillance of antibiotic resistance patterns among $S$. aureus strains should be considered in health programs.

\section{Acknowledgements}

None Declared.

\section{Financial Disclosure}

None Declared.

\section{Funding/Support}

This research was funded, in part, by an operating grant of the Dean of Research and Graduate Studies at the University of Isfahan, and a grant from Ministry of Health of Iran, Deputy of research and innovation. 


\section{Authors' Contribution}

None Declared.

\section{References}

1. Akpaka PE, Monecke S, Swanston WH, Rao AC, Schulz R, Levett PN Methicillin sensitive Staphylococcus aureus producing PantonValentine leukocidin toxin in Trinidad \& Tobago: a case report. J Med Case Rep. 2011;5:157.

2. Gordon RJ, Lowy FD. Pathogenesis of methicillin-resistant Staphylococcus aureus infection. Clin Infect Dis. 2008;46 Suppl 5:S350-9.

3. Diep BA, Chambers HF, Graber CJ, Szumowski JD, Miller LG, Han LL, et al. Emergence of multidrug-resistant, community-associated, methicillin-resistant Staphylococcus aureus clone USA300 in men who have sex with men. Ann Intern Med. 2008;148(4):249-57.

4. Zetola N, Francis JS, Nuermberger EL, Bishai WR. Communityacquired meticillin-resistant Staphylococcus aureus: an emerging threat. Lancet Infect Dis. 2005;5(5):275-86.

5. Turlej A, Hryniewicz W, Empel J. Staphylococcal cassette chromosome mec (Sccmec) classification and typing methods: an overview. Pol J Microbiol. 2011;60(2):95-103.

6. Wax RG. Bacterial resistance to antimicrobials. CRC. 2008.

7. Deurenberg RH, Stobberingh EE. The molecular evolution of hospital- and community-associated methicillin-resistant Staphylococcus aureus. Curr Mol Med. 2009;9(2):100-15

8. Aligholi M, Emaneini M, Jabalameli F, Shahsavan S, Dabiri H, Sedaght H. Emergence of high-level vancomycin-resistant Staphylococcus aureus in the Imam Khomeini Hospital in Tehran. Med Princ Pract. 2008;17(5):432-4.

9. Howden BP, Davies JK, Johnson PD, Stinear TP, Grayson ML. Reduced vancomycin susceptibility in Staphylococcus aureus, including vancomycin-intermediate and heterogeneous vancomycin-intermediate strains: resistance mechanisms, laboratory detection, and clinical implications. Clin Microbiol Rev. 2010;23(1):99-139.

10. Styers D, Sheehan DJ, Hogan P, Sahm DF, Laboratory-based surveillance of current antimicrobial resistance patterns and trends among Staphylococcus aureus: 2005 status in the United States. Ann Clin Microbiol Antimicrob. 2006;5:2.

11. Klein E, Smith DL, Laxminarayan R. Hospitalizations and deaths caused by methicillin-resistant Staphylococcus aureus, United States, 1999-2005. Emerg Infect Dis. 2007;13(17):1840-6.

12. Kwon JC, Kim SH, Park SH, Choi SM, Lee DG, Choi JH, et al. Molecular epidemiologic analysis of methicillin-resistant Staphylococcus aureus isolates from bacteremia and nasal colonization at 10 intensive care units: multicenter prospective study in Korea. J Korean Med Sci. 2011;26(5):604-11.

13. Chen SY, Wang JL, Chen TH, Chiang WC, Wang JT, Chen SC, et al. Differences between methicillin-resistant Staphylococcus aureus bacteremic isolates harboring type IV and type V staphylococcal cassette chromosome mec genes based on prior patient healthcare exposure. Eur J Clin Microbiol Infect Dis. 2010;29(12):1539-46.

14. Laupland KB, Ross T, Gregson DB. Staphylococcus aureus bloodstream infections: risk factors, outcomes, and the influence of methicillin resistance in Calgary, Canada, 2000-2006. J Infect Dis. 2008;198(3):336-43.

15. Martinez JL. Antibiotics and antibiotic resistance genes in natural environments. Science. 2008;321(5887):365-7.

16. Popovich KJ, Weinstein RA, Hota B. Are community-associated methicillin-resistant Staphylococcus aureus (MRSA) strains replacing traditional nosocomial MRSA strains? Clin Infect Dis. 2008;46(6):787-94

17. Kateete DP, Kimani CN, Katabazi FA, Okeng A, Okee MS, Nanteza A, et al. Identification of Staphylococcus aureus: DNase and Mannitol salt agar improve the efficiency of the tube coagulase test. Ann Clin Microbiol Antimicrob. 2010;9:23.

18. Wikler MA. Performance standards for antimicrobial susceptibility testing: Sixteenth informational supplement. Clinical and Laboratory Standards Institute. 2006.

19. Methods for dilution antimicrobial susceptibility tests for bacteria that grow aerobically. Wayne (PA). 1993.

20. Jonas D, Speck M, Daschner FD, Grundmann H. Rapid PCR-based identification of methicillin-resistant Staphylococcus aureus from screening swabs. J Clin Microbiol. 2002;40(5):1821-3.

21. egel JD, Rhinehart E, Jackson M, Chiarello L, Committee HICPA Management of multidrug-resistant organisms in health care settings, 2006. American J Infect cont. 2007;35(2):165.

22. Japooni A, Alborzi A, Orafa F, Rasouli M, Farshad S. Distribution patterns of methicillin resistance genes (mecA) in Staphylococcus aureus isolated from clinical specimens. Iran Biomed J. 2004;8(4):173-78

23. Rahimi F, Bouzari M, Maleki Z, Rahimi F. Antibiotic susceptibility pattern among Staphylococcus spp. with emphasis on detection of mecA gene in methicillin resistant Staphylococcus aureus isolates. Iranian J Clin Infect Dis. 2009;4(3):143-50.

24. Saderi H, Owlia P, Jalali-Nadoushan MR. Difference in epidemiology and antibiotic susceptibility of methicillin resistant and methicillin susceptible Staphylococcus aureus isolates. Iranian J Clin Infect Dis. 2009;4(4):219-23.

25. Vahdani P, Saifi M, Aslani MM, Asarian AA, Sharafi K. Antibiotic resistant patterns in MRSA isolates from patients admitted in ICU and infectious ward. Tanaffos. 2004;3(11):37-44.

26. Arora S, Devi P, Arora U, Devi B. Prevalence of Methicillin-resistant Staphylococcus Aureus (MRSA) in a Tertiary Care Hospital in Northern India. J Lab Physicians. 2010;2(2):78-81.

27. Vidhani S, Mehndiratta PL, Mathur MD. Study of methicillin resistant S. aureus (MRSA) isolates from high risk patients. Indian J Med Microbiol. 2001;19(2):13-6.

28. Fluit AC, Wielders CL, Verhoef J, Schmitz FJ. Epidemiology and susceptibility of 3,051 Staphylococcus aureus isolates from 25 university hospitals participating in the European SENTRY study. $J$ Clin Microbiol. 2001;39(10):3727-32. 